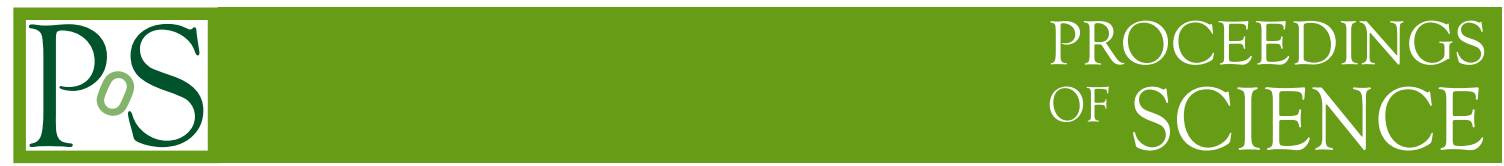

\title{
CP Violation and the Determination of the CKM
}

\section{Matrix}

\author{
Frank Porter*t \\ Physics Department 356-48 \\ Caltech \\ Pasadena, CA 91125 \\ E-mail: fcp@hep.caltech.edu
}

The current status of measurements of the elements of the CKM matrix is reviewed, with emphasis on recent results. The standard model continues to survive, but there are some interesting hints of issues that bear watching.

35th International Conference of High Energy Physics

July 22-28, 2010

Paris, France

\footnotetext{
* Speaker.

${ }^{\dagger}$ From the BABAR collaboration.
} 


\section{The CKM matrix}

The CKM matrix, $V$, relates mass and flavor eigenstates in the left-handed charged current couplings. It is convenient to express the matrix in the form of an expansion in powers of the parameter $\lambda$, which is roughly $1 / 5$ :

$$
V=\left(\begin{array}{lll}
V_{u d} & V_{u s} & V_{u b} \\
V_{c d} & V_{c s} & V_{c b} \\
V_{t d} & V_{t s} & V_{t b}
\end{array}\right)=\left(\begin{array}{ccc}
1-\frac{\lambda^{2}}{2} & \lambda & A \lambda^{3}(\rho-i \eta) \\
-\lambda & 1-\frac{\lambda^{2}}{2} & A \lambda^{2} \\
A \lambda^{3}(1-\rho-i \eta) & -A \lambda^{2} & 1
\end{array}\right)+O\left(\lambda^{4}\right)
$$

$C P$ violation comes from the complex nature of the matrix, parameterized here by $\eta$.

The CKM matrix is important because: (i) It is fundamental in the Standard Model (SM). For many predicitions in the standard model, we need to know the CKM matrix, which is a point in a four-dimensional parameter space. (ii) It is the source of $C P$ violation in the $\mathrm{SM}$, although apparently not the only actual source of $C P$ violation. (iii) It provides a framework to search for physics beyond the SM, via tests of unitarity, consistency tests of quantities related in the SM, and rare $C P$ violating processes in the SM. Due to space constraints, I limit the scope of the discussion to the theme of the CKM matrix in the SM, keeping the quest for new physics (NP) as an important undercurrent. We start by looking at the magnitudes of the elements, and then turn to the CPviolation quantities.

\section{The CKM matrix: magnitudes of elements}

Table 1 summarizes the present knowledge of the magnitudes of the CKM matrix elements, with the values from the 2008 Review of Particle Properties [1] for comparison. We briefly discuss the progress in each value. The magnitude $\left|V_{u d}\right|$ is best determined in superallowed nuclear beta decays. There continues to be progress here, and Hardy and Towner have recently completed a new comprehensive evaluation yielding $\left|V_{u d}\right|=0.97425 \pm 0.00022$ [2].

The value $\left|V_{u s}\right|$ continues to be best determined in kaon decays, either leptonic or $K_{l 3}$. The FlaviaNet Kaon Working group has recently completed an evaluation of the data, resulting in the average $\left|V_{u s}\right|=0.2253 \pm 0.0013$ [3]. Lattice calculations are used to evaluate the ratio of decay constants in the case of the leptonic decays, and to evaluate the form factor in the case of the $K_{l 3}$ decays [4]. This is the dominant uncertainty.

There is also a recent contribution to measuring $\left|V_{u s}\right|$ using tau decays. BABAR has measured the branching fractions for both $\tau \rightarrow K \ell v$ and $\tau \rightarrow \pi \ell v$. Taking the ratio yields [5]:

$$
\begin{aligned}
R_{K / \pi} & \equiv \frac{\mathscr{B}\left(\tau^{-} \rightarrow K^{-} v_{\tau}\right)}{\mathscr{B}\left(\tau^{-} \rightarrow \pi^{-} v_{\tau}\right)}=\frac{f_{K}^{2}\left|V_{u s}\right|^{2}\left(1-\frac{m_{K}^{2}}{m_{\tau}^{2}}\right)^{2}}{f_{\pi}^{2}\left|V_{u d}\right|^{2}\left(1-\frac{m_{\pi}^{2}}{m_{\tau}^{2}}\right)^{2}}\left(1-\delta_{L D}\right) \\
& =0.06531 \pm 0.00056 \pm 0.00093
\end{aligned}
$$

As in the case of the $K_{l 2}$ evaluation, the ratio of the kaon to pion branching fractions is used so that only the ratio of decay constants is needed. Using $f_{K} / f_{\pi}=1.189 \pm 0.007$ and $\delta_{L D}=0.0003 \pm$ 0.0044 , the result is: $\left|V_{u s}\right|=0.2255 \pm 0.0024$ [5], in good agreement with the kaon measurements. Inclusive $\tau \rightarrow s$ decays (in the form of a sum over exclusive decays) may also be used to determine 


$$
\begin{array}{ccc}
0.97425 \pm 0.00022 & 0.2253 \pm 0.0008 & 0.00392 \pm 0.00046 \\
(0.97418 \pm 0.00027) & (0.2255 \pm 0.0019) & (0.00393 \pm 0.00036) \\
& & \\
0.230 \pm 0.011 & 1.04 \pm 0.06 & 0.0409 \pm 0.0007 \\
(0.230 \pm 0.011) & (1.04 \pm 0.06) & (0.0412 \pm 0.0011) \\
& & \\
0.0081 \pm 0.0005 & 0.0387 \pm 0.0023 & 0.88 \pm 0.07 \\
(0.0081 \pm 0.0006) & (0.0387 \pm 0.0023) & \left(0.77_{-0.24}^{+0.18}\right)
\end{array}
$$

Table 1: Magnitudes of the CKM elements, showing the progress since 2008 (in parentheses [1]).

$\left|V_{u s}\right|$. A new, preliminary, evaluation has been performed with the present data [5], giving $\left|V_{u s}\right|=$ $0.2166 \pm 0.0023$, and an old discrepancy is actually getting worse, now to 3.6 sigma; this remains a mystery to resolve. My average, including the kaon and tau exclusive (but not inclusive $\tau \rightarrow s$ ) determinations, is $\left|V_{u s}\right|=0.2253 \pm 0.0008$.

The determination of $\left|V_{u b}\right|$ is difficult because of its small size. It is determined in both exclusive and inclusive semileptonic approaches. The main difficulty in the inclusive approach is the huge background from $b \rightarrow c$. To reduce the background, a selection on low $M_{X}$, the mass of the hadronic system in the $B \rightarrow X \ell v$ decay, or a selection on high $q^{2}$ (4-momentum transfer to the leptonic system) is usually made. However this then requires theoretical input to extrapolate to the whole distribution. A number of models are used for this. Averaging across models gives $\left|V_{u b}\right|=0.00441 \pm 0.00026$ (exp) \pm 0.00024 (thy) for the recent Belle analysis on $657 \mathrm{M} B \bar{B}$ events [6]. The corresponding $B A B A R$ preliminary inclusive result on its full dataset of $467 \mathrm{M} B \bar{B}$ events is $\left|V_{u b}\right|=0.00431 \pm 0.00035$ [7].

There are also recent results on $\left|V_{u b}\right|$ from exclusive $B \rightarrow u$ decays. The exclusive approach reduces the background problem, but at the cost of decreased statistics. In this approach one also needs to evaluate form factors from thoery, typically with Light Cone Sum Rules (LCSR) or lattice calculations. This becomes the dominant uncertainty in the result. The results for the Belle and $B A B A R$ branching fractions to $\pi \ell v$ are consistent (Belle [8]: $(1.49 \pm 0.04 \pm 0.07) \times 10^{-4}$; BABAR [9]: $\left.(1.41 \pm 0.05 \pm 0.07) \times 10^{-4}\right)$, as are the results for $\left|V_{u b}\right|$ (Table 2)

Purely leptonic measurements of $\left|V_{u b}\right|$ in $B \rightarrow \ell v$ decays are also possible, and there are new results from both Belle and $B A B A R$ on $B \rightarrow \tau v$ (the $\mu \nu$ and $e v$ channels are more strongly helicitysuppressed). The expected rate is small, and background suppression is difficult. The technique is to look for additional observed energy in the event; if only neutrinos are missed, this additional energy should be zero. The Belle and BABAR results are consistent (Table 2) and are rather large, though with large uncertainties.

The most recent measurements of $\left|V_{u b}\right|$ in all three approaches are shown in Table 2. A longstanding issue has been the difference between inclusive and exclusive results. This persists in recent measurements. For example, if we compare the Belle inclusive and exclusive measurements, the inclusive is 2.3 sigma higher than the exclusive. Averaging these results must be done with care, because of the correlations among the uncertainties. For now, the best available average is based on the end of 2009 HFAG compilation [12], from which the CKMfitter group obtains 


\begin{tabular}{lll} 
Measurement & Experiment & $V_{u b}$ \\
\hline & & \\
Inclusive & Belle [6] & $0.00441 \pm 0.00024$ \\
Inclusive & $B A B A R[7]$ & $0.00431 \pm 0.00035$ \\
Exclusive $\pi \ell v$ & Belle [8] & $0.00343 \pm 0.00033$ \\
Exclusive $\pi \ell v$ & $B A B A R[9]$ & $0.00295 \pm 0.00031$ \\
$B \rightarrow \tau \nu$ & Belle [10] & $0.00484 \pm 0.00079$ \\
$B \rightarrow \tau \nu$ & $B A B A R[11]$ & $0.0057 \pm 0.0019$
\end{tabular}

Table 2: Recent measurements of $V_{u b}$.

$\left|V_{u b}\right|=0.00392 \pm 0.00009 \pm 0.00045$ [13]

With the updated elements of the first row of the CKM matrix, we can make a check on unitarity. We compare the SM prediction ( $V$ is $3 \times 3$ unitary) with measurement:

$$
1=\left|V_{u d}\right|^{2}+\left|V_{u s}\right|^{2}+\left|V_{u b}\right|^{2}=0.99995 \pm 0.00057
$$

This test passes with flying colors. We can also obtain Bayesian limits on mixing with a possible 4th generation:

$$
\begin{aligned}
\left|V_{u 4}\right| & =\sqrt{1-\left|V_{u d}\right|^{2}+\left|V_{u s}\right|^{2}+\left|V_{u b}\right|^{2}} \\
& <0.031\left(90 \% \mathrm{CL}, \text { flat prior in }\left|V_{u 4}\right|^{2}\right) \\
& <0.061\left(90 \% \mathrm{CL}, \text { flat prior in }\left|V_{u 4}\right|\right)
\end{aligned}
$$

This limit is not very constraining, a fourth generation could contribute at the same level as the third generation. Using just the first two generations, the data are still not sufficiently precise to require the third generation.

The value of $\left|V_{c d}\right|$ is determined from charm production by neutrinos. In principle it can be determined in leptonic and semileptonic decays of the $D$ meson, but this needs theoretical input on the $D$ decay constant or on form factors. There is no recent change in $\left|V_{c d}\right|$ (Table 1), although theoretical progress continues (e.g., [14]). The value of $\left|V_{c s}\right|$ is obtained from leptonic $D_{s}$ decays, and from semileptonic $D$ decays to strangeness. The accuracy is limited by the theoretical uncertainties in the $D_{s}$ decay constant and form factors. Again, there is no recent update on this value (Table 1).

There has been substantial continued effort to measure $\left|V_{c b}\right|$, with new results from both Belle and $B A B A R$. As with $\left|V_{u b}\right|$, this matrix element is determined with both exclusive and inclusive approaches. Belle and $B A B A R$ present new results on $\left|V_{c b}\right|$ in exclusive $B$ to charm decays, $B \rightarrow D^{*} \ell v$ from Belle [15] and $B \rightarrow D \ell v$ from $B A B A R$ [16]. Theoretical input from lattice calculations is used to relate the branching fraction measurements to $\left|V_{c b}\right|$ through the form factors, yielding the reults in Table 2. There is also a recent $B_{A B} B R$ measurement of $\left|V_{c b}\right|$ in inclusive $B$ to charm [16]. The method involves determining the heavy quark parameters $\left(m_{b}, m_{c} / m_{b}\right.$, etc) in the non-perturbative corrections using OPE and the moments of distributions of hadronic mass and lepton energy. The result for $\left|V_{c b}\right|$ is shown in Table 2. As for $\left|V_{u b}\right|$, the inclusive results tend to be higher than the exclusive. It seems plausible that there will eventually be a common explanation. Again, averaging must be done with care, and I propose the data through the end of 2009 [12], with the CKMfitter average as the best current estimate: $\left|V_{c b}\right|=0.04089 \pm 0.00038 \pm 0.00059$ [13]. 


\begin{tabular}{lll} 
Measurement & Experiment & $V_{c b}$ \\
\hline & & \\
Inclusive & $B A B A R[16]$ & $0.04205 \pm 0.00045 \pm 0.00070$ \\
Exclusive $D^{*} \ell v$ & Belle [15] & $0.0375 \pm 0.0015$ \\
Exclusive $D \ell v$ & $B A B A R[16]$ & $0.0392 \pm 0.0018 \pm 0.0013 \pm 0.0009$ (lattice)
\end{tabular}

Table 3: Recent measurements of $V_{c b}$.

The bottom row of the CKM matrix is the most poorly determined. The best value for $\left|V_{t d}\right|$ comes from $B$ mixing, in which the top quark contributes in the box diagrams. The uncertainty is dominated by lattice uncertainties in relating the observed mixing to $\left|V_{t d}\right|$. Since $\left|V_{t s}\right|$ is somewhat better determined from $B_{s}$ mixing, we may use the ratio of $\left|V_{t d}\right|$ to $\left|V_{t s}\right|$ (theory is more reliable for the ratio), plus the value of $\left|V_{t s}\right|$ to extract a value for $\left|V_{t d}\right|=0.0081 \pm 0.0005$.

There is another approach presented here [17]. The idea is to measure $\left|V_{t d} / V_{t s}\right|$ in the "inclusive" ratio of radiative $b \rightarrow d$ and $b \rightarrow s$ transitions. These are penguin diagram decays, so NP is possible in the loop. Hence this method is of interest as a search for NP. Instead of being truly inclusive, a sum of seven modes related by replacing a $d$ quark with an $s$ quark is used. For example, the $B \rightarrow \pi^{+} \pi^{-} \gamma$ and $B \rightarrow K^{+} \pi^{-} \gamma$ decays are included. The result is $\left|V_{t d} / V_{t s}\right|=$ $0.199 \pm 0.022$ (stat) \pm 0.024 (sys) \pm 0.002 (thy). The ratio is consistent with the B mixing result, so yields no evidence of NP.

As mentioned, the $\left|V_{t s}\right|$ element is determined from $B_{s}$ mixing, where the dominant uncertainty is from the lattice calculation. There is no recent update on $\left|V_{t s}\right|$ (Table 1). Finally, $\left|V_{t b}\right|$ has been measured by $\mathrm{CDF}$ and D0 in single top production, with the most recent combined result from 2009 of $\left|V_{t b}\right|=0.88 \pm 0.07$ [18]. This is an improvement over the 2008 lower limit of 0.74 (90\% C.L.) from this method, as well as the $0.77_{-0.24}^{+0.18}$ value from the precision electroweak fit with top loops in $Z \rightarrow b \bar{b}$ [1]. Given the measuremements of the other elements, $\left|V_{t b}\right|$ should be very close to 1 in the SM.

\section{The CKM matrix: $C P$ violation and angles of the unitarity triangles}

We turn now to $C P$ violation and the CKM matrix, the source of $C P$ violation in the SM. One manifestation of $C P$ violation is in the unitarity condition, for which products of different rows or columns express triangles in the complex plane. With $C P$ violation these triangles all have the same non-zero area in the SM. Two triangles in particular are the subject of current attention. The first, with commensurate sides, is what we usually mean when we say "unitarity triangle". The triangle relation is:

$$
0=V_{u d} V_{u b}^{*}+V_{c d} V_{c b}^{*}+V_{t d} V_{t b}^{*}=O\left(\lambda^{3}\right)+O\left(\lambda^{3}\right)+O\left(\lambda^{3}\right)
$$

It has angles $\alpha, \beta$, and $\gamma$, also known respectively as $\phi_{2}, \phi_{1}, \phi_{3}$. We'll look at the measurements of these angles. The other triangle is defined by the relation:

$$
0=V_{u s} V_{u b}^{*}+V_{c s} V_{c b}^{*}+V_{t s} V_{t b}^{*}=O\left(\lambda^{4}\right)+O\left(\lambda^{2}\right)+O\left(\lambda^{2}\right)
$$

It has $\beta_{s}$ as the angle between the two larger sides; $\beta_{s}$ should be small in the standard model. There are new results here as well, presented elsewhere [19] 

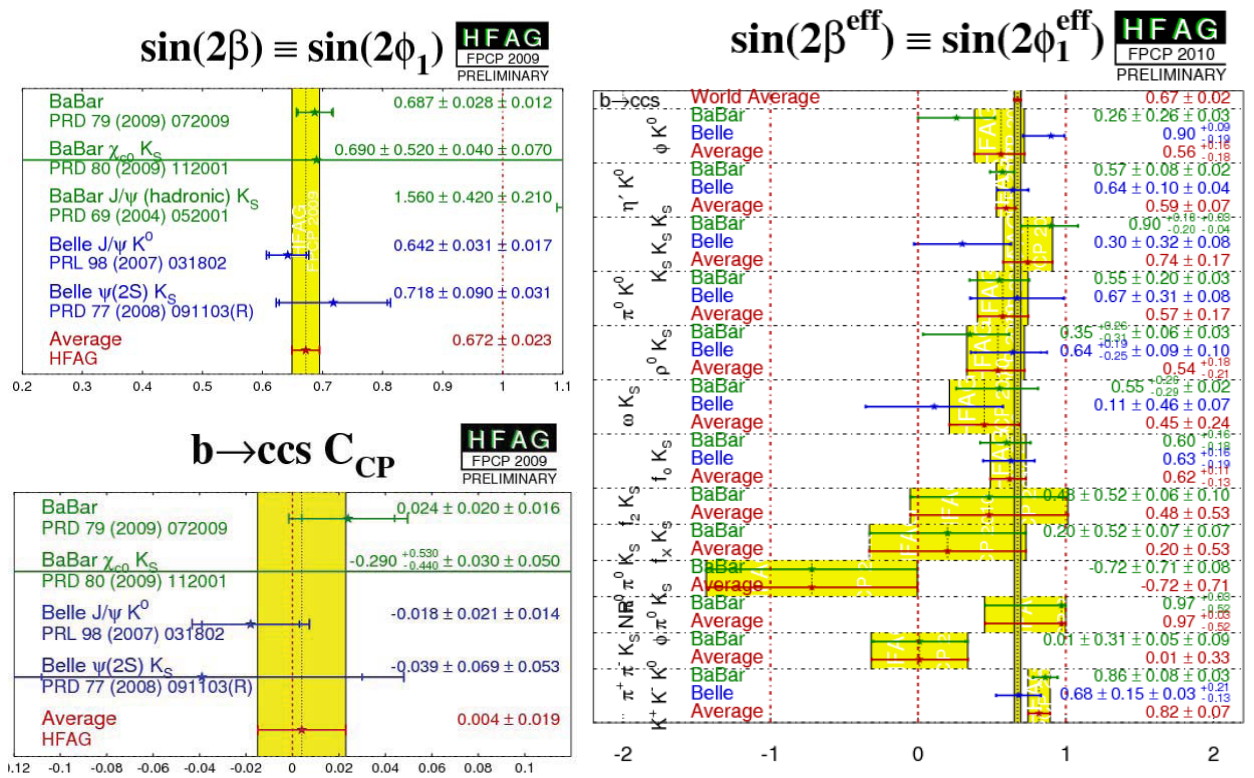

Figure 1: Left: Results on the sine and cosine terms in the time-dependent asymmetry for $b \rightarrow c \bar{c} s$ transitions; Right: Results on $\sin 2 \beta_{\text {eff }}$ for penguin modes. [12]

The angle $\beta / \phi_{1}$ is the one that started the asymmetric $B$ factory efforts. The $b \rightarrow c \bar{c} s$ transition, such as in $B \rightarrow J / \psi K_{S}$, is particularly clean both theoretically and experimentally, hence is called the "golden mode". $B A B A R$ [20] has published its golden mode results on its entire dataset: $\sin 2 \beta=$ $0.687 \pm 0.028 \pm 0.012$ (for zero $\cos \Delta m t$ dependence); Belle [21] is currently working on its final result, with an expected uncertainty on $\sin 2 \phi_{1}$ of 0.024 for $772 \mathrm{M} B \bar{B}$ events.

We may test the SM by comparing the golden mode result with results from penguin diagrams where NP may enter the loop. Belle [21] presented a time-dependent Dalitz plot analysis of the $b \rightarrow s s \bar{s}$ penguin mode $B^{0} \rightarrow K^{+} K^{-} K_{S}$, obtaining preferred solution:

$$
\begin{aligned}
\phi_{1}^{\text {eff }}\left(\phi(1020) K_{S}^{0}\right) & =(32.2 \pm 9.0 \pm 2.6 \pm 1.4(\mathrm{DP} \text { model }))^{\circ} \\
\phi_{1}^{\text {eff }}\left(f_{0}(980) K_{S}^{0}\right) & =(31.3 \pm 9.0 \pm 3.4 \pm 4.0(\mathrm{DP} \text { model }))^{\circ}
\end{aligned}
$$

The result is consistent with $\phi_{1}^{\text {eff }}=\phi_{1}$. Also, $B_{A B} B A$ presented preliminary branching fractions from the Dalitz plot analysis of $B^{0} \rightarrow \bar{D}^{0} \pi^{+} \pi^{-}$[22]. This channel may eventually provide another way to measure $\sin 2 \beta$ and $\cos 2 \beta$ in the time-dependent Dalitz plot analysis for $B^{0} \rightarrow D_{C P} \pi^{+} \pi^{-}$.

The results from the golden modes are summarized in Fig. 1, both for $\sin 2 \beta$ and for the coefficient of the $\cos \Delta m t$ term in the time-dependence. This coefficient is expected to be zero in the SM. The results are consistent with this. On the right is a comparison of several penguin modes with the $c \bar{c} s$ result. On this scale the $c \bar{c} s$ result is shown by a narrow interval. Again, the point of this is to look for deviations, indicating the possible presence of NP in the penguin loops. For the most part there is consistency, but there are a couple of roughly $2 \sigma$ deviations to watch. 
The second angle, $\alpha / \phi_{2}$, is a little trickier to measure. It is accessible in $b \rightarrow u \bar{u} d$ transitions, such as $B \rightarrow \pi^{+} \pi^{-}, \rho^{+} \rho^{-}, \pi^{+} \pi^{-} \pi^{0}, a_{1}^{ \pm} \pi^{\mp}$ but penguin graphs involving a different CKM phase are a source of "pollution". Fortunately, by measuring different isospin amplitudes, the desired tree amplitude may be recovered [23]. The existing results as averaged by CKMfitter and UTfit and used as inputs in their fits are:

$$
\begin{aligned}
\left(89.0_{-4.2}^{+4.4}\right)^{\circ} & \text { CKMfitter [13] } \\
(91.4 \pm 6.1)^{\circ} & \text { UTfit [24] }
\end{aligned}
$$

The angle $\gamma / \phi_{3}$ is the most difficult, indeed when we were first proposing the $B$ factories, we didn't know how to do this one. However, it is accessible in the interference between the $b \rightarrow c \bar{u} s$ and $b \rightarrow u \bar{c} s$ amplitudes, both $O\left(\lambda^{3}\right)$. A suitable pair of channels is $B^{-} \rightarrow D^{* 0} K^{-}$and $B^{-} \rightarrow \bar{D}^{* 0} K^{-}$, where interference is possible if the $D$ and $\bar{D}$ decay to a common final state. To measure $\gamma$, we then compare the $B^{-}$and $B^{+}$decays. Various approaches to such a measurement are being carried out, neglecting $D^{0} \bar{D}^{0}$ mixing, and several new results are presented.

In the first approach, referred to as "GLW" [25], we use $D$ and $\bar{D}$ decays to $C P$ eigenstates, such as $K^{+} K^{-}$or $K_{S} \pi^{0}$. They are both $V_{u s}$ Cabibbo suppressed. A new result with this method, based on $468 \mathrm{M} B \bar{B}$ at $B A B A R$, was presented at ICHEP [26]:

$$
\gamma(\text { degrees, } \bmod 180,68 \% \text { C.I. })=(11.3,22.7) \cup(80.9,99.1) \cup(157.3,168.7) \text {. }
$$

Another approach is the ADS method [27], using $D^{0} \rightarrow K^{+} \pi^{-}$and $\bar{D}^{0} \rightarrow K^{+} \pi^{-}$. In this case, the $D^{0}$ decay is doubly Cabibbo suppressed, while the $\bar{D}$ decay is Cabibbo allowed. A new result with this method, also with $468 \mathrm{M} B \bar{B}$ at $B A B A R$, results in a $68 \%$ confidence interval for $\gamma\left(\bmod 180^{\circ}\right)$ of approximately $27-152^{\circ}$ [26]. This region is larger than the GLW region on the same dataset.

Potentially the best results, but the most difficult analysis (referred to as GGSZ [28]) is to select 3-body $D$ decays and make a Dalitz plot analysis simultaneously for $B^{+}$and $B^{-}$to extract $\gamma$. Both Belle [29] and $B_{A} B_{A R}$ [26] present new results with this method. Both use $B^{ \pm} \rightarrow D^{(*)} K^{ \pm}$ decays, $B A B A R$ also includes $B^{ \pm} \rightarrow D K^{* \pm}$. The results from $B A B A R(468 \mathrm{M} B \bar{B})$ and Belle $(657 \mathrm{M} B \bar{B})$ are consistent:

$$
\begin{aligned}
\gamma / \phi_{S}(\bmod 180) & =\left[78.4_{-11.6}^{+10.8} \pm 3.6(\text { syst }) \pm 8.9(\text { model })\right]^{\circ} \text { Belle } \\
& =[68 \pm 14 \pm 4(\text { syst }) \pm 3(\text { model })]^{\circ} B A B A R
\end{aligned}
$$

In order to perform the Dalitz plot analysis for $\gamma$, a good understanding of the Dalitz plot is needed. So far, this is approached by assuming a model for the Dalitz plot and adjusting parameters to best fit the Dalitz plot for a large sample of decays. In particular, it is the differences in the strong phases that are needed as a function of position on the Dalitz plot. CLEO-c has shown that it is possible to measure these phase differences without resorting to a model, by making use of the quantum correlations at the $\psi(3770)$ [30]. The results are compared with the model used by $B A B A R$, finding good agreement, which is very encouraging.

In addition to the measurements of the unitarity triangle angles, several searches for new physics in $C P$ violation have been shown. These include searches in $B, D, K$, and $\tau$ decays [31]. For lack of space I can't discuss these, but I can summarize by saying that the SM has so far survived. 

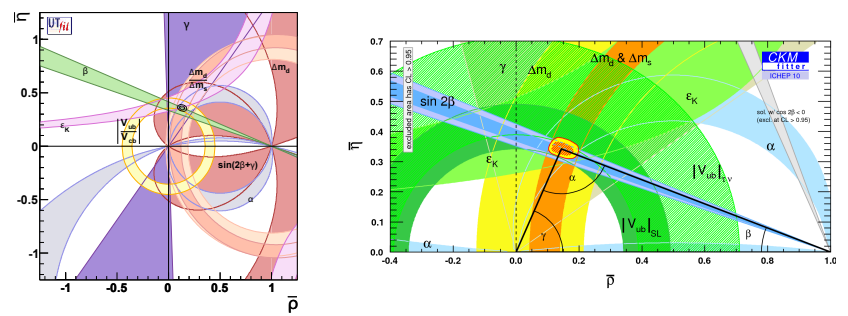

Figure 2: Bayesian (left [24]) and frequentist (right [13]) fits for the unitarity triangle.

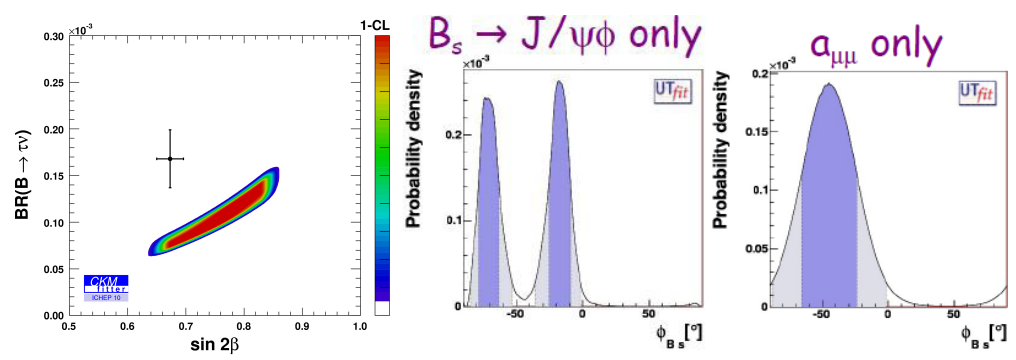

Figure 3: Left: CKMfitter plots probing the $B \rightarrow \tau v, \sin 2 \beta$ comparison with the SM [13]. The data point shows the measured values of $B \rightarrow \tau v, \sin 2 \beta$, compared with the result of the standard model fit from all other data as the band; Middle/Right: UTfit plots illustrating the difficulties presented by the new results on $\phi_{B_{S}}$ [24] (see text).

Finally, Fig. 2 shows the updated global unitarity triangle fits to the CKM data. On the left is the UTfit result [24], displayed in the $(\bar{\rho}, \bar{\eta})$ plane. On the right is the CKMfitter result [13] (where I have shown an expanded scale). The UTfit analysis is Bayesian and the CKMfitter is frequentist. The global consistency of the CKMfitter fit is at the 2 sigma level. In looking at the details of the fit, both groups identify two areas of discrepancy. First, both find a $2.6 \sigma$ discrepancy between the measured value of $\sin 2 \beta$ and the value from a fit excluding the measured value. Second, a 2.8 or $3.2 \sigma$ discrepancy is found for the value of the branching fraction for $B \rightarrow \tau \nu$. UTfit finds a third inconsistency, in $\varepsilon_{K}$ at the level of $1.7 \sigma$.

We can further characterize the two largest discrepancies. Figure 3, left, shows the branching fraction for $B \rightarrow \tau \nu$ plotted against $\sin 2 \beta$. The point with errors shows the direct measurements. The colored region shows the predicted position based on all the other data (95\% CL). In the $\rho, \eta$ plane, we find that the $B \rightarrow \tau \nu$ measurement breaks the $\sin 2 \beta$ band into two regions of high probability, with a dip in between, right where the other measurements prefer.

It has also been presented that there is another $3 \sigma$ effect in the D0 [19] dimuon analysis. An interesting plot (Fig. 3 middle/right) was made by the UTfit group showing $\phi_{B_{s}}\left(\arg \left(m_{12} / \Gamma_{12}\right)\right.$ for the $B_{S}$ system. On the left is the posterior from $B_{s} \rightarrow J / \psi \phi$ (not including new CDF result), and the right is the posterior from the like sign dimuon asymmetry. Both prefer large values. But it is amusing that the left plot has a dip where the right plot peaks.

Here are my conclusions, starting by referring back to Table 1 for the best current values of the magnitudes of the CKM matrix elements, not assuming unitarity. A fourth generation can easily fit 


$\begin{array}{ccc} & \text { CKMfitter [13] } & \text { UTfit [24] } \\ A & 0.812_{-0.027}^{+0.013} & 0.8095 \pm 0.0095 \\ \lambda & 0.22543 \pm 0.00077 & 0.22545 \pm 0.00065 \\ \bar{\rho} & 0.144 \pm 0.025 & 0.132 \pm 0.020 \\ \bar{\eta} & 0.342_{-0.015}^{+0.016} & 0.358 \pm 0.012 \\ \alpha\left(^{\circ}\right) & 91.0 \pm 3.9 & 87.8 \pm 3.0 \\ \sin 2 \beta & 0.689_{-0.021}^{+0.023} & 0.705 \pm 0.018 \\ \left.\gamma^{\circ}\right) & 67.2 \pm 3.9 & 69.8 \pm 3.0\end{array}$

Table 4: ICHEP 2010 averages (assuming $3 \times 3$ unitarity, SM)

with these numbers. Table 4 shows the best-fit values of the four Wolfenstein parameters, and then the three angles of the unitarity triangle. In general, the SM is very much alive and well. However, we have also seen some issues that we should watch seriously. Some of them will most likely be solved without requiring NP. These perhaps include the $\tau \rightarrow s$ puzzle and the inclusive/exclusive discrepancies. Some of the others have the potential to involve new physics (e.g., [32]), though it is premature to rule out more mundane explanations. We will have to wait to see whether we have the tip of an iceberg, or merely an ice cube melting away. There is much activity and many new results in this area. The prospects for future even more exciting results are very bright, with the LHC, the advances in kaons, the super B factories, and tau/charm factories.

\section{References}

[1] C. Amsler et al. (Particle Data Group), Phys. Lett. B667 (2008) 1.

[2] J. C. Hardy and I. S. Towner, Phys. Rev. C 79 (2009) 055502.

[3] FlaviaNet Kaon Working Group, arXiv:1005.2323 [hep-ph] (2010); see also F. Archilli, these proceedings.

[4] P. A. Boyle et al., Eur. Phys. C 69 (2010) 159.

[5] BABAR, A. Lusiani, these proceedings; B. Aubert at al., Phys, Rev, Lett, 105 (2010) 051602.

[6] Belle, P. Urquijo et al., Phys. Rev. Lett. 104 (2010) 021801.

[7] BABAR, M. Sigamani, these proceedings.

[8] Belle, H. Ha, these proceedings.

[9] BABAR, H. W. Wulsin, these proceedings; P. del Amo Sanchez et al., arXiv:1005.3288 [hep-ex] (2010).

[10] Belle, J. Stypuła, these proceedings.

[11] BABAR, G. De Nardo, these proceedings.

[12] Heavy Flavor Averaging Group (HFAG), http://www.slac.stanford.edu/xorg/hfag.

[13] CKMfitter, S. T'Jampens, these proceedings. CKMfitter Group (J. Charles et al.), Eur. Phys. J. C41, 1-131 (2005) [hep-ph/0406184], updated results and plots available at: http://ckmfitter.in2p3.fr; also A. Lenz et al., arXiv:1008.1593 [hep-ph].

[14] W. Lucha, D. Melikhov, and S. Simula, these proceedings; arXiv:hep-ph/1008.2951 (2010). 
[15] Belle, W. Dungel, these proceedings.

[16] BABAR, A. Petrella, these proceedings; B. Aubert et al., Phys. Rev. Lett., 104 (2010) 011802; B. Aubert et al., Phys. Rev. D 81 (2010) 032003.

[17] BABAR, D. Bard, these proceedings; P. del Amo Sanchez et al., Phys. Rev. D-RC 82 (2010) 051101.

[18] B. Quinn, these proceedings; arXiv:/0908.2171 [hep-ex] (2009).

[19] For a summary, see G. Borissov, these proceedings.

[20] BABAR, B. Aubert et al., Phys. Rev. D 79 (2009) 072009.

[21] Belle, T. Higuchi, these proceedings.

[22] BABAR, T. Latham, these proceedings.

[23] M. Gronau and D. London, Phys. Rev. Lett. 65 (1990) 3381.

[24] UTfit, C. Tarantino, these proceedings; http://www.utfit.org/.

[25] M. Gronau and D. Wyler, Phys. Lett. B265 (1991) 172; M. Gronau and D. London, Phys. Lett. B253 (1991) 483 .

[26] BABAR, F. Martinez-Vidal, these proceedings; P. del Amo Sanchez et al., Phys. Rev. D 82 (2010) 072004; P. del Amo Sanchez et al., Phys. Rev. D 82 (2010) 072006; P. del Amo Sanchez et al., Phys. Rev. Lett. 105 (2010) 121801.

[27] D. Atwood, I. Dunietz, and A. Soni, Phys. Rev. Lett. 78 (1997) 3257; Phys. Rev. D 63 (2001) 036005.

[28] A. Giri, Y. Grossman, A. Soffer, and J. Zupan, Phys. Rev. D 68 (2003) 054018.

[29] Belle, N. Joshi, these proceedings; A. Poluektov et al., Phys. Rev. D 81 (2010) 112002.

[30] CLEO-c, G. Wilkinson, these proceedings; R. Briere et al., Phys. Rev. D 80 (2009) 032002.

[31] Belle: T. Higuchi, these proceedings; H. Sahoo, these proceedings; B. Ko, these proceedings; M. Shapkin, these proceedings; BABAR: M. Bellis, these proceedings; CDF: M. Mattson, these proceedings; KEK E391a: H. Watanabe, these proceedings; NA48: A. Winhart, these proceedings.

[32] E. Lunghi and A. Soni, arXiv:hep-ph/1010.6069 (2010). 